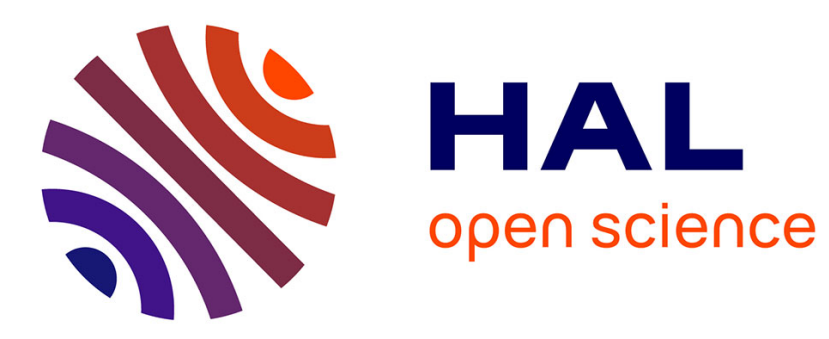

\title{
Scaling of susceptibility and the size of the critical region in an amorphous GdAl spin glass (invited)
}

\author{
A. P Malozemoff, Y. Imry, B. Barbara
}

\section{To cite this version:}

A. P Malozemoff, Y. Imry, B. Barbara. Scaling of susceptibility and the size of the critical region in an amorphous GdAl spin glass (invited). Journal of Applied Physics, 1982, 53 (11), pp.7672-7677. 10.1063/1.330179 . hal-01660278

\section{HAL Id: hal-01660278 https://hal.science/hal-01660278}

Submitted on 10 Dec 2017

HAL is a multi-disciplinary open access archive for the deposit and dissemination of scientific research documents, whether they are published or not. The documents may come from teaching and research institutions in France or abroad, or from public or private research centers.
L'archive ouverte pluridisciplinaire HAL, est destinée au dépôt et à la diffusion de documents scientifiques de niveau recherche, publiés ou non, émanant des établissements d'enseignement et de recherche français ou étrangers, des laboratoires publics ou privés. 


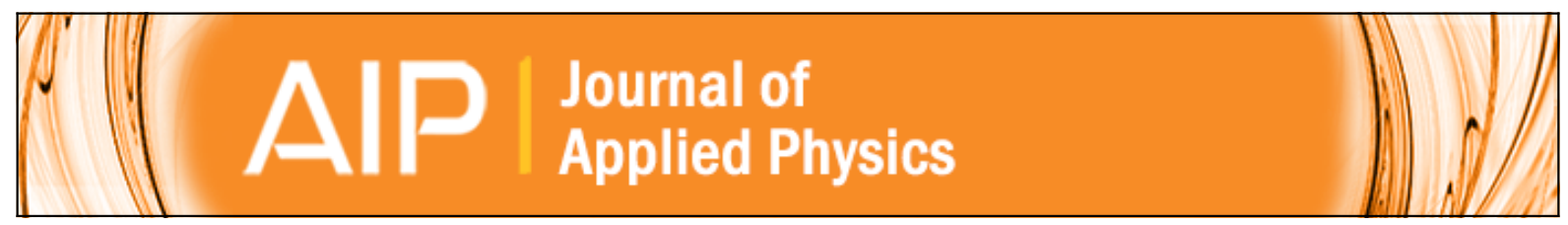

\section{Scaling of susceptibility and the size of the critical region in an amorphous GdAl spin glass (invited)}

A. P. Malozemoff, Y. Imry, and B. Barbara

Citation: Journal of Applied Physics 53, 7672 (1982); doi: 10.1063/1.330179

View online: http://dx.doi.org/10.1063/1.330179

View Table of Contents: http://scitation.aip.org/content/aip/journal/jap/53/11?ver=pdfcov

Published by the AIP Publishing

\section{Articles you may be interested in}

Magnetic correlations in an amorphous GdAl spin glass

J. Appl. Phys. 61, 3639 (1987); 10.1063/1.338698

Lowfield susceptibility of GdAl spin glass: Coolingrate dependence and field dependence of peak temperature

J. Appl. Phys. 57, 3389 (1985); 10.1063/1.335105

Chaotic spin glasses: An upper critical dimension (invited)

J. Appl. Phys. 55, 1646 (1984); 10.1063/1.333429

Further studies of nonlinear susceptibility of $\mathrm{GdAl}$ and $\mathrm{MnCu}$ spin glasses

J. Appl. Phys. 53, 2205 (1982); 10.1063/1.330818

Magnetic phase diagram of the GdAl and GdCu amorphous alloy systems

J. Appl. Phys. 49, 1689 (1978); 10.1063/1.324889

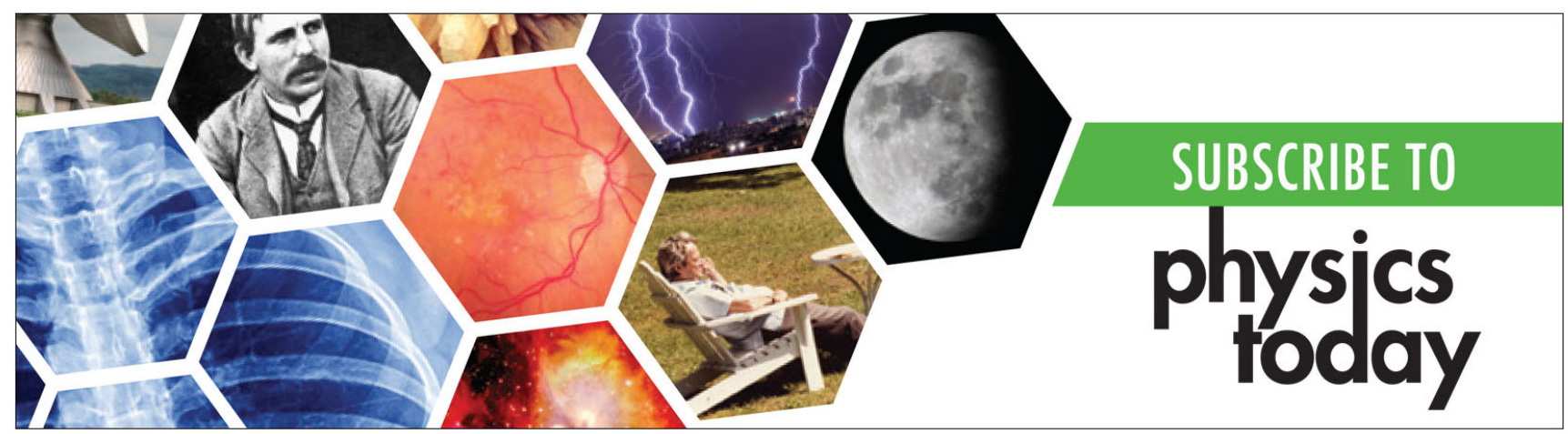




\title{
Scaling of susceptibility and the size of the critical region in an amorphous GdAI spin glass (invited)
}

\author{
A. P. Malozemoff and Y. Imry \\ T. J. Watson Research Center, Box 218, Yorktown Heights, New York 10598
}

B. Barbara

Laboratoire Louis Néel, CNRS, 166X, F-38042 Grenoble, France

\begin{abstract}
We extend our earlier SQUID magnetometry studies of the susceptibility of amorphous $\mathrm{Gd}_{37} \mathrm{~A}_{63}$ spin glass to a temperature range $5 \leq \mathrm{T} \leq 100 \mathrm{~K}$ and a field range $0.5 \leq \mathrm{H}<50000 \mathrm{Oe}$. This enables us to determine the size of the critical region around the spin glass transition at $\mathrm{T}_{\mathrm{G}}=15.5 \mathrm{~K}$; it extends to approximately $22 \mathrm{~K}$ in temperature and $15000 \mathrm{Oe}$ in field. We show that the range of reduced temperature $t=\left(T-T_{G}\right) / T_{G}$ for spin glass critical behavior is determined by the parameter $1 / Z^{1 / 2}$, where $Z$ is the number of interacting neighbors, in rough agreement with experiment, and that the field range can be estimated from a crossover relation $H \alpha t^{\phi / 2}$. We also find that nonanalyticity in $\chi(\mathrm{H})$ as $\mathrm{H} \rightarrow 0$ persists at all temperatures below $\mathrm{T}_{\mathrm{G}}$ and argue that this can cause apparent nonanalyticity above $T_{G}$ as well in the presence of sample inhomogeneity
\end{abstract}

PACS numbers: $75.30 . \mathrm{Kz}, 75.40 .-\mathrm{s}, 75.50 . \mathrm{Kj}$

In earlier papers (1-4) we have reported on SQUID magnetometer studies of spin glasses. There have been two main conclusions: One has been to confirm the lore that the DC field-cooled magnetization of a spin glass shows no time dependence and corresponds, therefore, to a thermal equilibrium magnetization. (1) The result conflicts with extrapolations of earlier frequency-dependent AC susceptibility data to low frequencies and it shows that kinetic models based on such AC susceptibility data break down in the long-time regime. The second result has been to investigate the field-dependent susceptibility in the DC equilibrium limit (2) and to show that it can be scaled (3-4) in field $\mathrm{H}$ and reduced temperature $t=\left(T-T_{G}\right) / T_{G}$, where $T_{G}$ is the transition temperature.

In this paper, we extend such a scaling analysis to test the various scaling relations and to investigate the limits of the scaling region both above and below the transition temperature $T_{G}$ of an amorphous $\mathrm{GdAl}$ spin glass. Although critical exponents have been determined by many authors in the past, (5-24) ours is the most complete analysis of the critical behavior of a spin glass reported so far and the first to determine boundaries of the critical region in both $\mathrm{H}$ and $\mathrm{T}$. However, we do not explicitly explore the possibility of a line of phase transitions in the $H, T$ plane except at $\mathbf{H}=\mathbf{0}$. Such a line has been predicted theoretically on the basis of mean field models $(16,25)$ and investigated experimentally. $(21,26)$ To what degree these ideas are compatible with our approach is a topic for further study.

\section{EXPERIMENTAL TECHNIQUES}

To investigate the size of the critical region, we extend our previous SQUID magnetometer study of the fieldcooled magnetization of an amorphous thin-film sample of $\mathrm{Gd}_{37} \mathrm{Al}_{63}$ to both higher and lower temperatures and fields. Data from $5 \mathrm{~K}$ up to $100 \mathrm{~K}$ and from 10 Oe up to $45 \mathrm{KOe}$ was taken in a SHE VTS-50 SQUID magnetometer. Data from $0.5 \mathrm{Oe}$ to $10 \mathrm{Oe}$ was taken in a non-commercial SQUID magnetometer, (27) whose field was provided by a solenoid in a Dewar shielded down to of order $1 \mathrm{mOe}$ from the earth's or other stray magnetic fields. Such data could not be taken in the SHE magnetometer because at low fields inhomogeneities invariably occur from trapped flux in the superconducting magnet. As pointed out earlier (28) inhomogeneities in the field cause an effective AC component as the sample is cycled between the counterwound pickup coils and therefore give rise to a spurious signal. We found that $x$ tended to be reduced in the presence of inhomogeneity and that this effect occurred only below the susceptibility cusp, where the spurious change in $\chi$ increased in magnitude with decreasing temperature. We monitored inhomogeneity by checking the symmetry of the SQUID output as a function of sample position and only include data where this inhomogeneity is less than $2 \%$. This criterion limits our data to a lowest field of $0.5 \mathrm{Oe}$. A hint of the effects of inhomogeneity appears in Fig. 1 and 2 of our earlier published data (reference 2), where the lowest field data crosses below higher field data at the lowest 
temperature. Fortunately, this effect was small in the temperature range we previously studied, but for the results reported here we must guard against it more carefully.

We have previously discussed the reasons for our normalization procedure to determine the field (4). In this study, we have directly used the field read-out of the VTS50 to determine the field from 45 down to $10 \mathrm{kOe}$. From 5 $\mathrm{kOe}$ down to $300 \mathrm{Oe}$ we have normalized at $80 \mathrm{~K}$ to the 10 kOe data at $80 \mathrm{~K}$. From 200 Oe down to 10 Oe we have normalized at $30 \mathrm{~K}$ to the $300 \mathrm{Oe}$ data at $30 \mathrm{~K}$. And from 10 Oe down to $0.5 \mathrm{Oe}$ (this section taken in our noncommercial magnetometer), we have normalized at $20 \mathrm{~K}$ to the $10 \mathrm{Oe}$ data at $20 \mathrm{~K}$. Since these normalizations assume linearity of $\mathrm{M}(\mathrm{H})$ at the normalization temperature over the given field range, linearity must be consistent within experimental error with the scaling relation that we are attempting to establish; this can be confirmed for the crucial $20 \mathrm{~K}$ normalizations from Eq. 6 and the fitting parameters given below.

In extending the data to lower temperatures, we have determined the paramagnetic signal from the substrate and have shown that its contribution to the nonlinear susceptibility of the whole sample can be ignored for our $0.2 \%$ accuracy. We have also checked demagnetizing effects and shown they are negligible for our thin film sample with field in the film plane. However, we have substracted a small ferromagnetic component $\mathrm{M}_{\mathrm{F}}$ which was detected by plotting $M$ vs. $H$ at $20 \mathrm{~K}$ below 20 Oe and determining the intercept. We treat $\mathrm{M}_{\mathrm{F}}$ by assuming it is constant at all temperatures. Even if it varies drastically with temperature, it gives no effect at low temperatures where $\chi$ happens to be very close to the value at the normalization temperature of $20 \mathrm{~K}$, and at worst $\chi\left(0.5 \mathrm{Oe}, \mathrm{T}_{\mathrm{G}}\right)$ may be decreased by $2 \%$. Finally, we have had to make a minor adjustment for difference in temperature calibration between the two apparatuses. These factors and greater experimental inaccuracy make the low field susceptibility data accurate to only about $\pm 1 \%$, whereas, the data for $\mathbf{H}>10 \mathrm{Oe}$ is accurate to about $\pm 0.2 \%$.

\section{RESULTS AND POWER LAW FITS}

Some results for $\chi \equiv \mathbf{M} / \mathbf{H}$ for slow field-cooling are shown in Fig. 1 as a function of temperature. Several sets of data (points) as a function of field are shown in linear

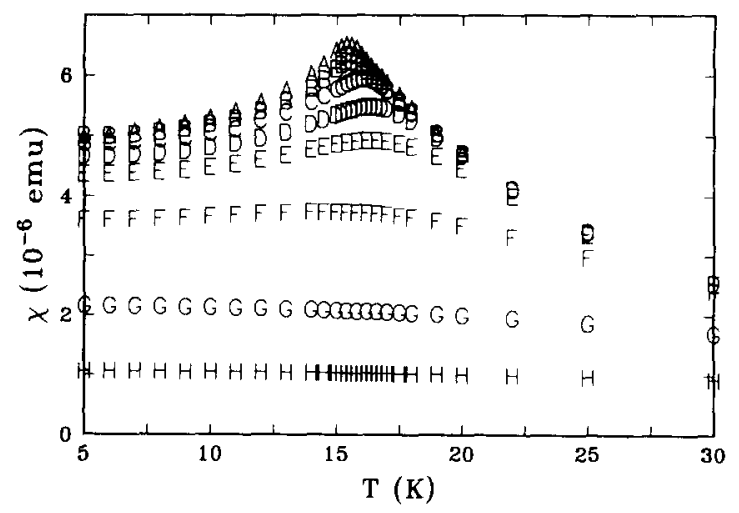

Fig. 1 Susceptibility $\chi=\mathbf{M} / \mathbf{H}$ of amorphous $\mathrm{Gd}_{37} \mathrm{Al}_{63}$ sample, $3.7 \mu \mathrm{m}$ thick, $7 \times 10^{-5} \mathrm{~cm}^{3}$, as a function of temperature $\mathrm{T}$ for the fields (in $\mathrm{Oe})$ : $\mathrm{A}(0.5), \mathrm{B}(5), \mathrm{C}(33), \mathrm{D}(158)$, $E(488), F(2061), G(10030), H(45010)$. plots in Fig. 2 and semi-logarithmic plots in Fig. 3. (Solid line fits will be explained below.) As in previous work $(3,4)$ we first fit the data to the empirical form

$$
\chi=\chi_{o}-\chi_{1} H^{p},
$$

where $\chi_{0}, \chi_{1}$ and $\mathrm{p}$ are functions of temperature. Although we could make fits with field ranges as small as from 0.5 to $50 \mathrm{Oe}$, we found that the fitting parameters were strongly influenced by scatter in the low field points and, therefore, looked very erratic when plotted versus temperature. As the field range of the fits increased, the parameters stabilized, giving excellent fits shown as solid lines in Fig. 3. As we increased the field range even further, the quality of the fits tended to decline.

Therefore, in Fig. 4 we choose to plot the parameters $\chi_{0}, \chi_{1}$ and $\mathrm{p}$ as determined from the field range $0<\mathrm{H}<$ 2000 Oe. Above $30 \mathrm{~K}, \chi_{1}$ and $\mathrm{p}$ could not be accurately determined because the changes in $\chi$ in the given field range were too small. The results agree qualitatively with our previous work, showing an anomaly in $\chi_{1}$ which is far stronger than the anomaly in $\chi_{0}$. The peak of $\chi_{0}$ and $\chi_{1}$ is at $15.5 \pm 0.1 \mathrm{~K}$, which defines $\mathrm{T}_{\mathrm{G}}$ for our subsequent analysis. Quantitatively, there are some small differences from our earlier work because of the lower field data, which show that our earlier extrapolations (3) for $\chi_{0}$ were on the low side. Correspondingly, we now find values of $p$, dipping to a minimum of 0.35 at $T_{G}$ while previously (3) we had reported a minimum of 0.5 .
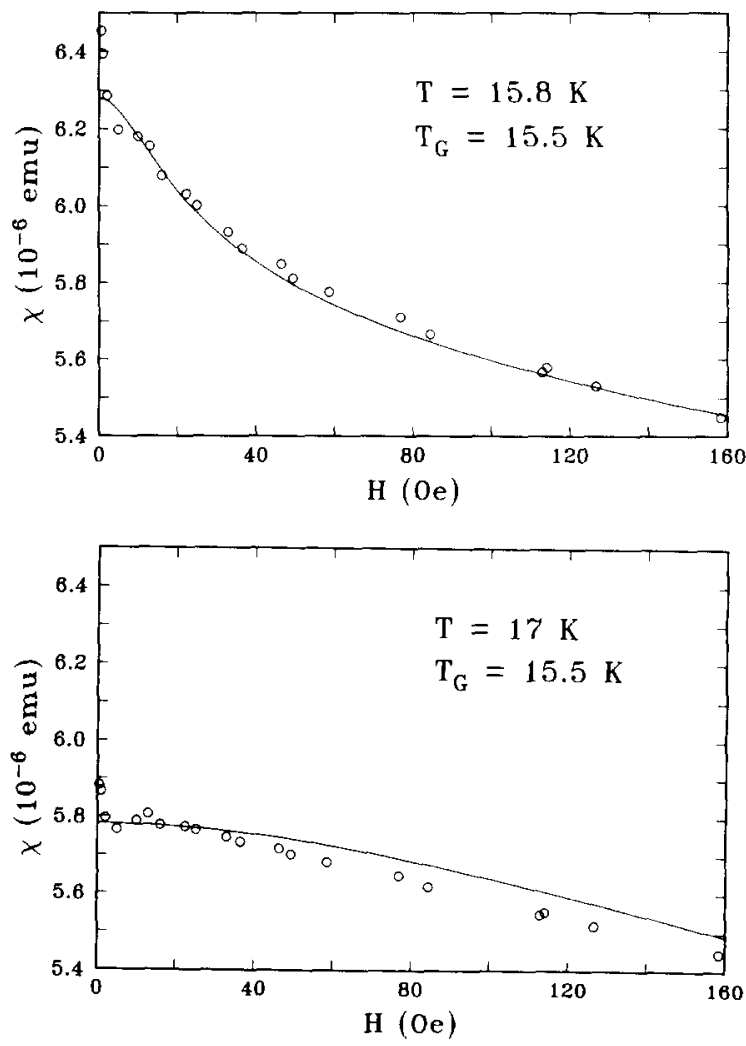

Fig. 2.

Measured susceptibility $\chi$ (circles) of GdAl sample vs. field $\mathrm{H}$ at two temperatures. Solid line is a fit according to parametrized scaling relation Eq. 8 and fitting parameters in text. 

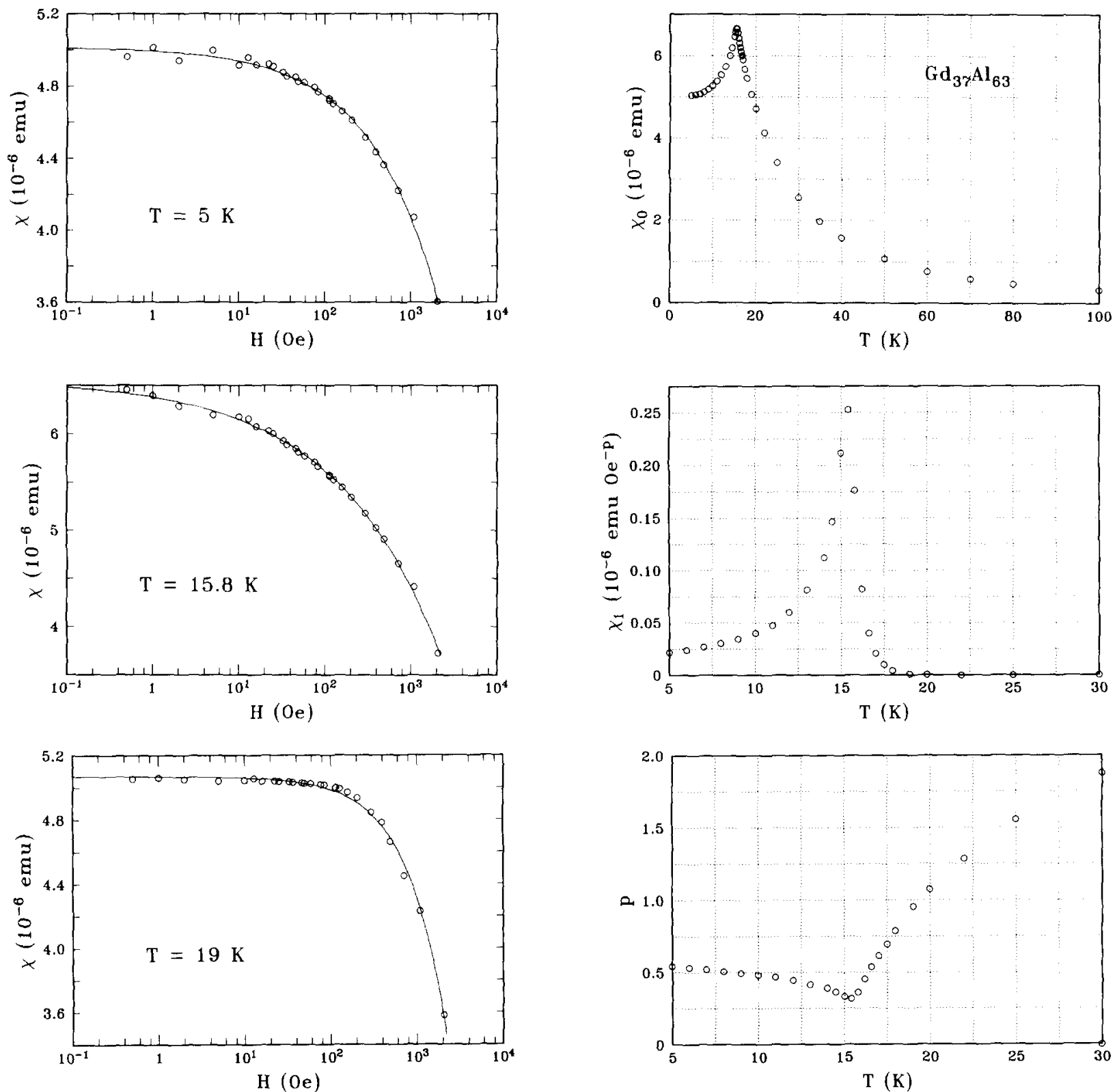

Fig. 3

Measured susceptibility $\chi$ (circles) of GdAl sample vs. field $\mathrm{H}$ for three temperatures. Solid line is a fit according to Eq. 1.

Using a mean field model, Toulouse and Gabay (15) had predicted a value 1 for $p$ at $T_{G}, 2$ above $T_{G}$ and $4 / 3$ below. Our new values are even further from their meanfield predictions than our earlier results. However, we do confirm their qualitative prediction of non-analytic behavior at all $\mathrm{T}$ below $\mathrm{T}_{\mathrm{G}}$. For the exponent $\mathrm{p}$ in Fig. $4 \mathrm{c}$ stays well below 1, becoming essentially temperature independent and equal to 0.55 at low temperatures. More mysterious are the values of $p$ below 2 above $T_{G}$ where one might expect regular $\mathrm{H}^{2}$ behavior; we will return to this question shortly.

\section{SCALING FITS}

We have previously suggested $(3,4)$ that a more consistent treatment of the data above $T_{G}$ can be obtained using a scaling relation

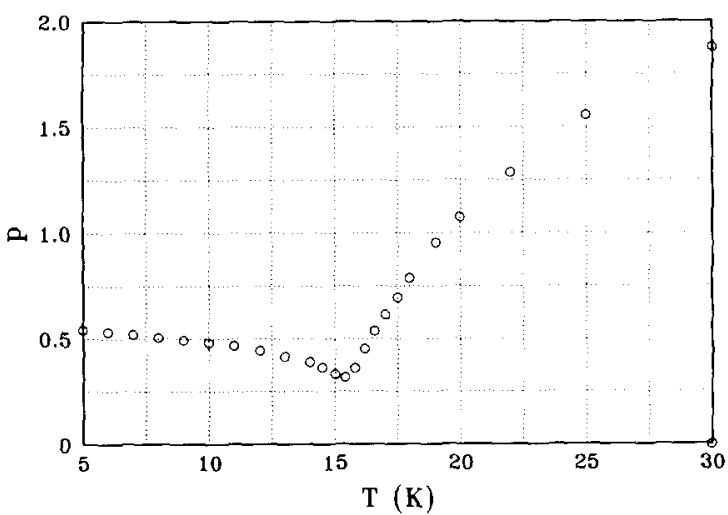

Fig. 4 Fitted parameters $\chi_{0}, \chi_{1}$ and $p$ of Eq. 1 as a function of temperature. The field range 0 to 2000 Oe was used for these fits.

$$
\begin{gathered}
\chi_{o}-\chi \equiv \chi_{s}=H^{2 / \delta} f\left(t / H^{2 / \phi}\right), \\
f(x) \rightarrow \text { const, } x \rightarrow o, \\
f(x)=x^{-\gamma}, x \rightarrow \infty,
\end{gathered}
$$

where $f$ is a scaling function to be determined, $t$ is reduced temperature $\left(T-T_{G}\right) / T_{G}, \chi_{S}$ is the "nonlinear" susceptibility, and $\delta, \gamma$ and $\phi$ are critical exponents. If the behavior is to be regular $\left(\chi_{s} \sim H^{2}\right)$ above $\mathrm{T}_{\mathrm{G}}$, these exponents must be related by

$$
\phi=\gamma \delta /(\delta-1) .
$$


Our previous treatment was not self-consistent because we determined $\chi_{o}$ from Eq. 1, which does not agree with Eq. 2. Here we attempt a self-consistent treatment by scaling the full $\chi$ without subtracting $\chi_{0}$. This becomes practical if we can paramatize $\chi_{0}$ and $f$. For $\chi_{0}$ we use the form

$$
\chi_{o}=\chi_{p}-A t^{\beta},
$$

where $\chi_{p}$ is the peak value of $\chi$ at $\mathrm{T}_{G}$ and $\mathrm{H}=0$, and $\mathrm{A}$ and $\beta$ are constants independent of temperature in the critical region. Suzuki (8) derived a scaling relation which showed that $\beta$ is, in fact, just the critical exponent of the spin glass order parameter. According to Chalupa, (7) $\beta$ is also given by the scaling relation.

$$
\beta=\gamma /(\delta-1) \text {. }
$$

Mizoguchi et al. (6) have also determined $\beta$ from a linear extrapolation of $\chi_{o}\left(T>T_{G}\right)$ to the region $\mathrm{T}<\mathrm{T}_{\mathrm{G}}$ and a comparison with $\chi_{0}\left(T<T_{G}\right)$. They obtained a value of 0.85 \pm 0.05 , and we confirm the result approximately with our new data. It should be noted, however, that a linear extrapolation of $\chi_{o}$ from above $T_{G}$ is difficult to justify if $\beta<1$ and, therefore, one should regard the precise value with some caution.

To parametrize the scaling function $f(x)$ we use the simplest possible form which correctly reproduces the two limits $\mathrm{x}=0$ and $\mathrm{x} \rightarrow \infty$ of Eq. 2:

$$
\chi_{s}=1 /\left[B\left(t^{\gamma} / H^{2}\right)+C H^{-2 / \delta}\right], t>o
$$

where $\mathbf{B}$ and $\mathrm{C}$ are constants independent of temperature in the critical region and where we have built in regular behavior at low $\mathbf{H}$ by using Eq. (3) to relate $\phi$ to $\gamma$ and $\delta$. This function closely simulates the form of the scaling function found in our previous work. $(3,4)$ The form indicates a crossover between regular $\left(\mathrm{H}^{2}\right)$ temperature-dependent behavior at low $\mathrm{H}$ and irregular $\left(H^{2 / \delta}\right)$ temperatureindependent behavior at high $\mathrm{H}$ (which of course is only meant to apply in the critical region). The crossover line in the $\mathrm{H}, \mathrm{T}$ plane is described by the equation

$$
H=(B / C)^{\phi / 2 \gamma} t^{\phi / 2} \text {, }
$$

obtained by setting the two terms in the denominator of Eq. (6) equal and using Eq. (3). Since we shall find that $\phi$ is significantly greater than 2 , this equation predicts that the field-region where one can observe normal $\mathrm{H}^{2}$ behavior becomes rapidly smaller as $t \rightarrow 0$. If this H-region is comparable to or smaller than our lowest measuring field, the fits to Eq. (1) would appear to show irregular $\chi(H)$ behavior with $\mathrm{p}<2$ above $\mathrm{T}_{\mathrm{G}}$, as observed. But there is an additional possible reason for this effect, namely, the sample inhomogeneity to be discussed below.

Combining Eqs. (4) and (6), we arrive at a parametrized form for $\chi$ in the critical region.

$$
\chi(H, t)=\chi_{p}-A t^{\beta}-\left[B\left(t^{\gamma} / H^{2}\right)+C H^{-2 / \delta}\right]^{-1}, t>0,
$$

where the 7 unknown constants $\chi_{p}, A, B, C, \beta, \gamma$ and $\delta$ are independent of temperature. Our procedure is to determine these constants from a nonlinear least-squares computer fit to the data. Although there are so many parameters, we have a sufficient number of data points $\left(\sim 500\right.$ above $\left.T_{G}\right)$ to obtain a well defined fit for all the parameters using data in an appropriate range of temperature and field. We have tried different ranges and find, not surprisingly, that the fitting parameters depend on the range.

The results of such a fit for the range $0 \leq \mathrm{H} \leq 2000$ Oe and $15.6 \leq T \leq 18 \mathrm{~K}$ give $\beta=0.93 \pm 0.04$, $\gamma=2.7 \pm 0.1, \delta=5.7 \pm 0.2, \chi_{p}=(6.42 \pm 0.02) \times 10^{-6} \mathrm{emu}$ $A=(5.64 \pm 0.04) \times 10^{-6}$ ema $K^{-\beta}, B=(3.5 \pm 0.1) \times 10^{13}$ $\mathrm{emu}^{-1} \mathrm{~K}^{-\gamma} \mathrm{Oe}^{2}$ and $\mathrm{C}=(6.9 \pm 0.6) \times 10^{6} \mathrm{emuOe}^{2 / \delta}$. The value for $\beta=0.93 \pm 0.04$ is in good agreement with the independent determination by Mizoguchi et al. (6) (and ourselves) of $\beta=0.85 \pm 0.05$, which provides one successful test of the scaling relations. Another test comes from Eq. (5) which, given $\gamma$ and $\delta$, yields $\beta=0.57 \pm 0.04$, substantially below the other two determinations. However, the error bars reflect only statistical and not systematic errors.

Next, in Fig. 5a, we plot $\chi_{\mathrm{s}}$, defined by

$$
\chi_{\mathrm{s}}=\chi_{\mathrm{p}}-\mathrm{At}^{\beta}-\chi \text {, }
$$
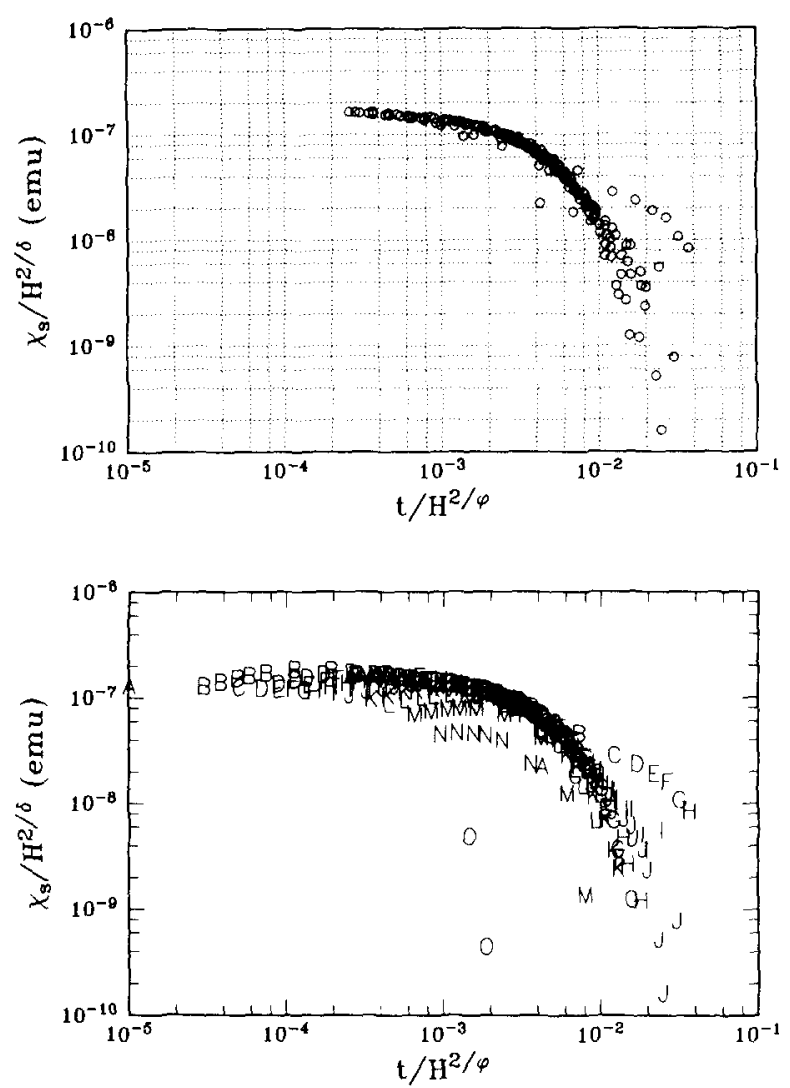

Fig. 5 Scaling fits of $x_{S}$ (from Eq. 9) divided by $\mathrm{H}^{2 / \delta}$, vs. $\mathrm{t} / \mathrm{H}^{2 / \phi}$. In a) the points come from the range $15.6 \leq \mathrm{T} \leq 18 \mathrm{~K}$ and $0<\mathrm{H}<2000$ Oe, for which the least squares fit to Eq. 8 was done $\left(T_{G}=15.5 \mathrm{~K}\right)$. In $b$ ), all positive points in the range $15.5 \leq \mathrm{T} \leq 100$ and $0<\mathrm{H}<$ $50000 \mathrm{Oe}$ are included, but the fitting parameters of Eq. 8 are the same as in a). The letters correspond to temperatures (in $\mathrm{K}$ ): $\mathrm{A}(15.6)$, $\mathrm{B}(15.8), \mathrm{C}(16), \mathrm{D}(16.2), \mathrm{E}(16.4), \mathrm{F}(16.6)$, $\mathrm{G}(16.8), \mathrm{H}(17), \mathrm{I}(17.5), \mathrm{J}(18), \mathrm{K}(19), \mathrm{L}(20)$, $\mathrm{M}(22), \mathrm{N}(25)$ and $\mathrm{O}(30)$. 
(where $x$ is as usual the experimental value), divided by $\mathrm{H}^{2 / \delta}$, vs. $\mathrm{t} / \mathrm{H}^{2 / \phi}$, where $\phi=3.3 \pm 0.4$ from Eq. (3). The shape of this function is similar to our previous result, $(3,4)$ as indeed it must be since we have built the shape into our fitting function Eq. (8). Some differences are the somewhat modified parameters and exponents and an increased scatter in the large $\mathrm{t} / \mathrm{H}^{2 / \phi}$ region. These effects are largely due to the new low field points of our present data base.

Actually, many of the low field points are completely missing from this plot because they give values of $\chi_{\mathrm{s}}$ (Eq. 9) which are negative and which can, therefore, not appear on a $\log -\log$ plot. Further insight into this problem can be obtained from Fig. 2, where solid lines show the predicted behavior for $\chi$ from Eq. 8 using the above fitting parameters. We see that, in fact, the fits fail to reproduce the sharp upturns in $\chi$ at very low fields. This is troubling because this is just the region where one might expect scaling behavior to be most valid. One is tempted to conclude that some kind of irregular $\chi(\mathrm{H})$ behavior persists above $\mathrm{T}_{\mathrm{G}}$, which might imply the existence of some other higher transition temperature to the regular $\left(x_{\mathrm{s}} \sim \mathrm{H}^{2}\right)$ paramagnetic state. There is also the possibility of crossover to some other kind of scaling behavior dominated, for example, by anisotropy or long-range magnetostatic forces.

However, we tentatively propose an alternative, less exotic explanation in terms of sample inhomogeneity. We have previously shown (1) the existence of macroscopic inhomogeneity in this sample, which is presumably responsible for the smearing of the susceptibility peak by at least $0.5 \mathrm{~K}$. And we can plausibly imagine that the tails of this distribution extend several degrees to either side of the average $T_{G}(15.5 \mathrm{~K})$. Now in a conventional phase transition, such as a ferromagnetic one, the critical point occurs at only one temperature; so smearing rapidly masks any nonanalytic critical behavior. However, in our spin-glass case, we have concluded from our data that there is a nonanalytic behavior of $\chi(\mathrm{H})$ around $\mathrm{H}=0$ at all temperatures below $T_{G}$. Smearing cannot mask such nonanalytic behavior so easily because the effect is cumulative; all sections of the sample with local $T_{G}$ 's above the average will contribute to the $\chi(\mathrm{H})$ divergence above that average. Since the nonanalytic behavior is quite strong, only a small portion of the sample need have its local $T_{G}$ above a given temperature to give the kind of low field upturns shown in Fig. 2.

We have earlier suggested the possibility of an intrinsic width to the transition in 3 dimensions, due to intrinsic fluctuations. (1) This would give rise to experimental smearing as well. However, this mechanism would imply analyticity in $\chi(\mathrm{H})$ at low fields, which we do not see down to 0.5 Oe. From this value and the crossover relation Eq. 7, we can estimate the maximum temperature broadening from this mechanism to be $0.03 \mathrm{~K}$, which is insufficient to explain the observed broading and reinforces the likelihood that sample inhomogeneity is the dominant mechanism.

Because of this effect, we cannot reduce the fieldrange of our nonlinear fits very far without running the risk of weighting the low-field data points too heavily. On the other hand, if we include too large a field and temperature range, we may exceed the critical region. The fit shown in Fig. 5a, taken with points in the range $15.5<\mathrm{T}<18 \mathrm{~K}$ and $0<\mathrm{H}<2000 \mathrm{Oe}$, appears to be roughly optimal in having enough points $(\sim 200)$ for a good fit while remaining well within the critical region.

\section{SIZE OF THE CRITICAL REGION}

To determine the size of the critical region, we now

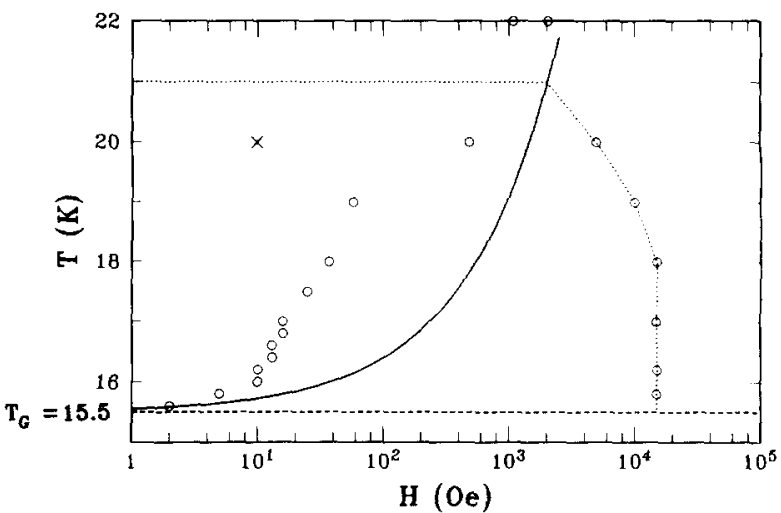

Fig. 6

Circles indicate limits of critical region in $\mathrm{H}, \mathrm{T}$ plane, as determined in text. Solid line is plot of crossover relation (Eq. 7) using fitting parameters given in text. Dotted line indicates hypothetical boundary of critical region, including region masked by inhomogeneity ef fect. Cross indicates normalization point for low field data $(<10$ Oe $)$.

plot all 500 data points above $\mathrm{T}_{\mathrm{G}}$, that is, points in the range $15.5<\mathrm{T}<100 \mathrm{~K}$ and $0<\mathrm{H}<50000 \mathrm{Oe}$, using the previous fitting parameters. Using Eq. 9 to determine $\chi_{\mathrm{s}}$, we obtain the scaling plot shown in Fig. $5 \mathrm{~b}$, where the letters label the different temperatures as listed in the figure caption. Now of course there are many points which scatter of the scaling curve of Fig. 5a, and these points are presumably the ones which fall outside the critical region. For example, the points at $25 \mathrm{~K}$, labeled by the letter $\mathrm{N}$ in Fig. $5 b$ never approach the scaling curve, whereas, some points of the letter $M$ just overlap the curve. In particular, the M-point at $2.5 \times 10^{-3}$ on the abscissa and $7 \times 10^{-8}$ on the ordinate corresponds to $22 \mathrm{~K}$ and 2000 Oe. We take this to be one boundary point of the critical region. Proceeding in this way, we construct the boundary shown in Fig. 6. It must be admitted that because we use an "eyeball" criterion for determining where the points deviate from the scaling curve, the boundary is only approximate, with at least factor of 2 error bars in field. However, uncertainties in the data and in the many fitting parameters do not easily permit a more quantitative treatment.

Considering now the shape of the boundary in Fig. 6, we argue that the dropoff in the low-field, high-temperature corner is an artifact of the low-field susceptibility "upturn", caused by sample inhomogeneity as discussed before. Thus, we conclude that the critical region extends essentially from 0 to about 15000 Oe in field and from 0 to about $(22 / 15.5)-1=0.4$ in reduced temperature. It is substantially larget than the region of our least squares fit, as we had hoped. Such a critical region is also very large by ferromagnetic standards where the critical region of threedimensional systems is typically of order $\Delta t \sim 0.1$ or less, depending on the range of the exchange.

We believe such a large critical region is plausible for a spin glass, as we suggest by the following argument: Consider a ferromagnet with $\mathrm{Z}$ interacting neighbors each coupling to a given spin with a ferromagnetic exchange interaction $E_{i j}=-2 J S_{i} \cdot S_{i}$. The ordering energy per spin is $Z J$, and in a mean field model, the ordering temperature is $\mathrm{T}_{\mathrm{F}} \sim \mathrm{ZJ} / \mathrm{k}$. It has been argued (29) that the relative size of the critical region is roughly determined by the fluctuation 
in energy $J$ (per spin) caused by a single spin flip, hence by the small parameter $J / Z J=1 / Z$. A parallel argument can be made for a spin glass with $\mathrm{Z}$ interacting neighbors each coupling to a given spin with a statistical distribution of interactions $E_{i j}= \pm 2 J S_{i} \cdot S_{j}$. Using the mean field_result (30) that the ordering energy per spin goes as $\sqrt{\mathrm{ZJ}}$, we arrive at the small parameter $1 / \sqrt{Z}$ as determining the critical region. Thus, for a given $\mathrm{Z}$, the spin glass critical region will be larger than the corresponding ferromagnetic critical region. Since typical ferromagnetic critical regions in three dimensions with nearest neighbor interactions have $\Delta \mathrm{T} / \mathrm{T}_{\mathrm{G}} \sim 0.1$, our result for a spin glass of $\sim 0.4$ is not implausible.

We can go a step further and relate the width in temperature to the width in field, via the crossover relation of Eq. 7, which yields the relative instability at $T_{\mathfrak{c}}$ to thermal and magnetic field perturbations (31). This is plotted as a solid line in Fig. 6. Substituting $t=0.4$ and the fitting parameters, we predict a field width $\mathrm{H}$ of $2500 \mathrm{Oe}$. Considering the uncertainties in the parameters and particularly in the boundary of the critical region, this prediction is in acceptable agreement with experiment. It shows that the critical region is amazingly large in field, much larger than early researchers might have guessed from the rapid rounding of their AC susceptibility cusps with applied field (32). Physically we believe the reason for the large size of the critical region along the field axis is that a uniform applied field is not directly conjugate to the spin glass order parameter (as it is to the ferromagnetic order parameter), and therefore it does not act as effectively to quench critical fluctuations. Of course, the fact that the critical region is so broad in both temperature and field makes it possible for us to determine it in spite of the large smearing of the susceptibility peak and the uncertainty of our data at low fields.

In summary, there is good evidence for the adequacy of the scaling treatment of the susceptibility of our amorphous GdAl spin glass. The critical exponents $\beta \sim 1, \gamma \sim 2.7, \delta \sim 5.7$, coupled with appropriate magnitude factors, suffice to correlate a large amount of data over a range of reduced temperatures from 0 to 0.4 and over a range of fields from 0 to $15000 \mathrm{Oe}$. These ranges are themselves plausible in terms of rough physical arguments. However, there are several danger signals. One scaling relation (Eq. 5) is poorly satisfied, and low field upturns fail to be described by the scaling. We have suggested transition broadening due to sample inhomogeneity as a possible cause of at least the latter problems, but a study of a sample with a sharper cusp would be the cleanest way to settle this issue. The possibility that the transition is intrinsically smeared at three dimensions is not ruled out but shown to be unlikely. Further discussion of the low temperature data is also deferred to later work. We remark in this connection that the nonanalytical behavior for all $\mathrm{T}<$ $T_{G}$ is a remarkable effect and certainly suggests a distinct spin glass phase, if not a line of critical points, below $T_{G}$.

The authors thank L. Krusin-Elbaum for essential assistance in the use of her fine SQUID magnetometer, $R$. J. Gambino for the sample, R. R. McGuire for assistance with the SHE magnetometer, and D. Mukamel, L. Kadanoff, E. Pytte and S. Kirkpatrick for useful discussions.

Y. Imry on leave of absence from the Department of Physics and Astronomy, Tel Aviv University, Tel Aviv, Israel.

\section{REFERENCES}

(1) A. P. Malozemoff and Y. Imry, Phys. Rev. B 24, 489 (1981).

(2) B. Barbara, A. P. Malozemoff and Y. Imry, Physica 108B, 1289 (1981).

(3) B. Barbara, A. P. Malozemoff and Y. Imry, Phys. Rev. Lett. 47, 1852 (1981).

(4) A. P. Malozemoff, B. Barbara and Y. Imry, J. Appl. Phys. 53, 2205 (1982).

(5) S. Katsura, Prog. Theor. Phys. 551049 (1976).

(6) T. Mizoguchi, T. R. McGuire, S. Kirkpatrick and R. J. Gambino, Phys. Rev. Lett. 38, 89 (1977), and Physica 86-88B, 783 (1977).

(7) J. Chalupa, S. S. Comm. 22, 315 (1977); 24, 429 (1977).

(8) M. Suzuki, Progr. Theor. Phys. 58, 1151 (1977).

(9) Y. Miyako, S. Chikazawa, T. Saito and Y. G. Yuochunas, J. Appl. Phys. 46, 1951 (1979); 52, 1779 (1981); Y. Miyako, S. Chikazawa, T. Sato and T. Saito, J. Magnetism and Magnetic Materials 15, 139 (1980). (10)M. J. Simpson, J. Phys. F9, 1377 (1979).

(11) J. J. Hauser, F. S. L. Hsu, G. W. Kammlott and J. V. Waszczak, Phys. Rev. B20, 339 (1979).

(12) S. Nagata, P. H. Keesom and H. R. Harrison, Phys. Rev. B29, 1633 (1979).

(13) K. Wada and H. Takayama, Prog. Theor. Phys. 64, 327 (1980).

(14) Y. Ueno and T. Oguchi, Prog. Theor. Phys. 63342 (1980).

(15) G. Parisi, and G. Toulouse, J. Phys. (Paris) Lett. 41, L361 (1980).

(16) G. Toulouse and M. Gabay, J. Phys. Lett. 92, L103 (1981).

(17) J. Vannimenus, G. Toulouse and G. Parisi, J. de Physique, (Paris) 42, 565 (1981).

(18) K. Honda and H. Nakano, Prog. Theor. Phys. 6583 and 95 (1981).

(19) C.A.M. Mulder, A. J. van Duyneveldt and J. A. Mydosh, Phys. Rev. B23, 1384 (1981).

(20) P. Monod and H. Bouchiat, J. de Physique Lett. (Paris), 43, L45.

(21) A. Berton, J. Chaussy, J. Odin, R. Rammal and R. Tournier, J. de Physique Lett. (Paris) 43, L153 (1982).

(22) S. Fujiki and S. Katsura, Prog. Theor. Phys. 65, 1130 (1981).

(23) F. Tanaka, Phys. Rev. B24, 6675 (1981).

(24) M. A. Moore and A. J. Bray, J. Phys. C15, L301 (1982).

(25) J. R. L. de Almeida and D. J. Thouless, J. Phys. A11, 983 (1978).

(26) R. V. Chamberlin, M. Hardiman, L. A. Turkevich and R. Orbach, Phys. Rev. B., to be published.

(27) L. Krusin-Elbaum, Ph.D. Thesis, New York University, 1979.

(28) D. Fields, L. Krusin-Elbaum and S. J. Williamson, Bull. Am. Phys. Soc. 25, 176 (1980).

(29) R. Brout, Phase Transitions, (Benjamin, New York, 1964).

(30) D. Sherrington and S. Kirkpatrick, Phys. Rev. Lett. 35, 1792 (1975); D. Sherrington, J. Phys. C 8, L208 (1975).

(31) F. J. Wegner, in Phase Transitions and Critical Phenomena vol. 6, C. Domb and M. S. Green, eds. (Academic Press, London, 1976).

(32) V. Cannella and J. A. Mydosh, Phys. Rev. B6, 4220 (1972). 